\title{
Charged Particle in a Flat Box with Static Electromagnetic Field and Landau's Levels
}

\author{
Gustavo V. López, Jorge A. Lizarraga \\ Departamento de Física, Universidad de Guadalajara, Guadalajara, México \\ Email:gulopez@cencar.udg.mx,jorge.a.lizarraga.b@gmail.com
}

How to cite this paper: López, G.V. and Lizarraga, J.A. (2020) Charged Particle in a Flat Box with Static Electromagnetic Field and Landau's Levels. Journal of Modern Physics, 11, 1731-1742.

https://doi.org/10.4236/jmp.2020.1110106

Received: July 26, 2020

Accepted: October 25, 2020

Published: October 28, 2020

Copyright $\odot 2020$ by author(s) and Scientific Research Publishing Inc. This work is licensed under the Creative Commons Attribution International License (CC BY 4.0).

http://creativecommons.org/licenses/by/4.0/

\begin{abstract}
We study the quantization of a charged particle motion without spin inside a flat box under a static electromagnetic field. Contrary to Landau's solution with constant magnetic field transverse to the box and using Fourier transformation, we found a full solution for the wave function which is different from that one given by Landau, and this fact remains when static electric field is added. However, the Landau's levels appear in all cases.
\end{abstract}

\section{Keywords}

Landau's Levels, Quantum Hall Effect, Flat Box

\section{Introduction}

The work of Klitzing, Dora and Pepper [1] presented a breakthrough in experimental physics due to the success in measuring the Hall voltage of a two-dimensional electron gas realized in a MOSFET. The important fact discovered in this experiment was that the Hall resistance is quantized, and Landau' eigenvalues solution [2] (Landau's levels) of a charged particle in a flat surface with magnetic field has become of great importance in trying to understand integer hall effect [1] [3] [4] [5] [6], fractional Hall effect [6] [7] [8] [9], and topological insulators [10]-[16]. These elements promise to become essential for future nanotechnology devices [17] [18] [19]. Due to this considerable application of the Landau's levels, it is worth to re-study this problem and its variations with an static electric field. In this paper, we solve the problem of a charged particle inside a flat box with lengths $L_{x}, L_{y}$, and $L_{z}$ such that $L_{z} \ll L_{x}, L_{y}$ by using the Fourier transformation, for three different cases: for a transverse constant magnetic field, for a constant magnetic field orthogonal to a constant electric field, and for a constant magnetic field parallel to a constant electric field. We show that there exists 
a different solution for this type of eigenvalue quantum problems than that one given by Landau, but having the same Landau's levels. We consider that this result could be relevant because Landau's solution is kept using in different works like Prange's [20], Laughling's [21], solid state and quantum transport books as well [3] [7] [22] [23].

\section{Analytical Approach for the Case $B=(0,0, B)$}

Let us consider a charged particle " $q$ " with mass " $m$ " in the box with a constant magnetic field orthogonal to the flat surface, $\boldsymbol{B}=(0,0, B)$, as shown in Figure 1 .

For a nonrelativistic charged particle, the Hamiltonian of the system (units CGS) is

$$
H=\frac{(\boldsymbol{p}-q \boldsymbol{A} / c)^{2}}{2 m},
$$

where $\boldsymbol{p}$ is the generalized linear momentum, $\boldsymbol{A}$ is the magnetic potential such that $\boldsymbol{B}=\nabla \times \boldsymbol{A}$, and " $\boldsymbol{C}$ " is the speed of light. We can choose the Landau's gauge to have the vector potential of the form $\boldsymbol{A}=(-B y, 0,0)$. Therefore, the Hamiltonian has the following form

$$
H=\frac{\left(p_{x}+q B y / c\right)^{2}}{2 m}+\frac{p_{y}^{2}}{2 m}+\frac{p_{z}^{2}}{2 m} .
$$

To quantize the system, we need to solve the Schrödinger's equation [24]

$$
i \hbar \frac{\partial \Psi}{\partial t}=\left\{\frac{\left(\hat{p}_{x}+q B y / c\right)^{2}}{2 m}+\frac{\hat{p}_{y}^{2}}{2 m}+\frac{\hat{p}_{z}^{2}}{2 m}\right\} \Psi .
$$

where $\Psi=\Psi(\boldsymbol{x}, t)$ is the wave function, $\hbar$ is the Plank's constant divided by $2 \pi, \hat{p}_{i}$ is the momentum operators such that $\left[x_{i}, \hat{p}_{j}\right]=i \hbar \delta_{i j}$. Now, the argument used by Landau is that due to commutation relation $\left[\hat{p}_{x}, \hat{H}\right]=0$, between the operators $\hat{p}_{x}$ and the Hamiltonian $\hat{H}$ (implying that $\hat{p}_{x}$ is a constant of motion), it is possible to replace this component of the momentum by $\hbar k_{x}$,

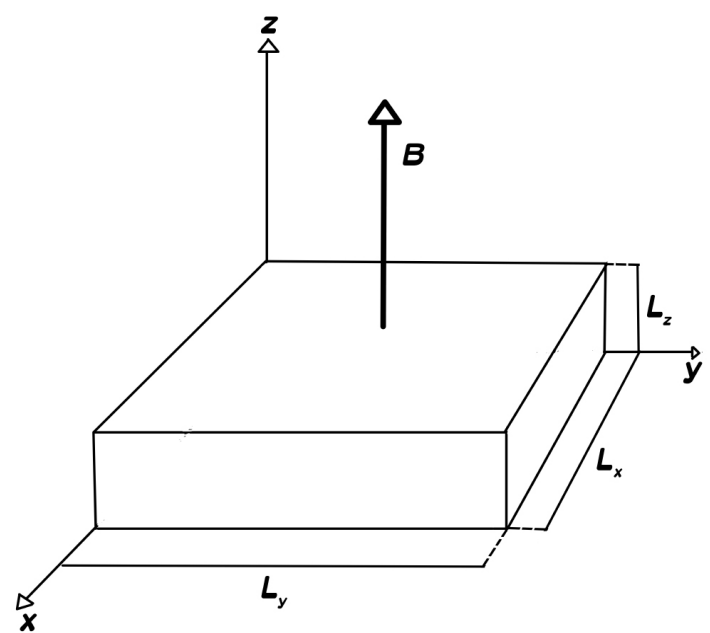

Figure 1. Electric charged in a flat box with magnetic field. 
having a solution for the eigenvalue problem of separable variable type, $f_{2}(x) f_{3}(y) f_{4}(z)$ for the resulting eigenvalue problem, once the time variable is separated. However, this problem can be fully addressed using the Fourier transformation. First, since the Hamiltonian $\hat{H}$ does not depend explicitly on time, the proposition

$$
\Psi(\boldsymbol{x}, t)=\mathrm{e}^{-i E t / \hbar} \Phi(\boldsymbol{x})
$$

reduces the equation to an eigenvalue problem

$$
\hat{H} \Phi=E \Phi .
$$

Then, this equation is written as

$$
\left\{\frac{1}{2 m}\left(\hat{p}_{x}^{2}+\frac{2 q B}{c} y \hat{p}_{x}+\frac{q^{2} B^{2}}{c^{2}} y^{2}\right)+\frac{\hat{p}_{y}^{2}}{2 m}+\frac{\hat{p}_{z}^{2}}{2 m}\right\} \Phi=E \Phi .
$$

The variable " $z$ " is separable through the proposition

$$
\Phi(\boldsymbol{x})=\phi(x, y) \mathrm{e}^{-i k_{z} z}, \quad k_{z} \in \Re,
$$

resulting in the following equation

$$
\left\{\frac{1}{2 m}\left(\hat{p}_{x}^{2}+\frac{2 q B}{c} y \hat{p}_{x}+\frac{q^{2} B^{2}}{c^{2}} y^{2}\right)+\frac{\hat{p}_{y}^{2}}{2 m}\right\} \phi=E^{\prime} \phi,
$$

where $E^{\prime}$ is

$$
E^{\prime}=E-\frac{\hbar^{2} k_{x}^{2}}{2 m}
$$

That is, the resulting partial differential equation is of the form

$$
\frac{1}{2 m}\left\{-\hbar^{2} \frac{\partial^{2} \phi}{\partial x^{2}}-i \frac{2 q B \hbar}{c} y \frac{\partial \phi}{\partial x}+\frac{q^{2} B^{2}}{c^{2}} y^{2} \phi\right\}-\frac{\hbar^{2}}{2 m} \frac{\partial^{2} \phi}{\partial y^{2}}=E^{\prime} \phi .
$$

This equation will be solved using Fourier transformation [25] on the variable " $x$ ",

$$
\hat{\phi}(k, y)=\mathcal{F}[\phi]=\frac{1}{\sqrt{2 \pi}} \int_{\Re} \mathrm{e}^{i k x} \phi(x, y) \mathrm{d} x .
$$

Applying Fourier transformation to this equation, knowing its property $\mathcal{F}[\partial \phi / \partial x]=(-i k) \hat{\phi}$, we get the ordinary differential equation

$$
-\frac{\hbar^{2}}{2 m} \frac{\mathrm{d}^{2} \hat{\phi}}{\mathrm{d} y^{2}}+\frac{m}{2} \omega_{c}^{2}\left(y-y_{0}\right)^{2} \hat{\phi}=E^{\prime} \hat{\phi}
$$

where $\omega_{c}$ is the cyclotron frequency

$$
\omega_{c}=\frac{q B}{m c}
$$

and $y_{0}=y_{0}(k)$ is the displacement parameter

$$
y_{0}=\frac{\hbar c}{q B} k .
$$

This equation is just the quantum harmonic oscillator in the " $y$ " direction displaced by an amount $y_{0}$. So, its solution in the Fourier' space is 


$$
\hat{\phi}_{n}(k, y)=\psi_{n}(\xi), \quad \xi=\sqrt{\frac{m \omega_{c}}{\hbar}}\left(y-y_{0}\right), \quad \psi_{n}(\xi)=A_{n} \mathrm{e}^{-\xi^{2}} H_{n}(\xi),
$$

being $H_{n}(\xi)$ the Hermit polynomials, and $A_{n}$ is a constant of normalization, $A_{n}=\left(m \omega_{c} / \pi \hbar\right)^{1 / 4} / \sqrt{2^{n} n !}$. and

$$
E_{n}^{\prime}=\hbar \omega_{c}(n+1 / 2) \text {. }
$$

Now, the solution in the real space $\phi_{n}(x, y)$ is gotten by using the inverse Fourier transformation [25],

$$
\phi_{n}(x, y)=\mathcal{F}^{-1}\left[\phi_{n}(k, y)\right]=\frac{1}{\sqrt{2 \pi}} \int_{\mathfrak{R}} \mathrm{e}^{-i k x} \psi_{n}\left(\sqrt{\frac{m \omega_{c}}{\hbar}}(y-\hbar c k / q B)\right) \mathrm{d} k .
$$

Making the change of variable $\sigma=\sqrt{m \omega_{c} / \hbar}(y-\hbar c k / q B)$, and knowing that the Fourier transform of the harmonic oscillator solution is another harmonic oscillator solution, we get

$$
\phi_{n}(x, y)=\frac{-q B}{\sqrt{m c^{2} \hbar \omega_{c}}} \mathrm{e}^{-i \frac{q B}{\hbar c} x y} \psi_{n}\left(\frac{q B x}{\sqrt{m c^{2} \hbar \omega_{c}}}\right) .
$$

This last equation is indeed a nonseparable solution of (8). Therefore, the normalized eigenfunctions and the eigenvalues of the eigenvalue problem (5) are (ignoring the sign)

$$
\Phi_{n, k_{z}}(\boldsymbol{x})=\frac{\sqrt{q B}}{\left(L_{y}^{2} L_{z}^{2} m c^{2} \hbar \omega_{c}\right)^{1 / 4}} \mathrm{e}^{-i\left(\frac{q B}{\hbar c} x y-k_{z} z\right)} \psi_{n}\left(\frac{q B x}{\sqrt{m c^{2} \hbar \omega_{c}}}\right) .
$$

and

$$
E_{n, k_{z}}=\hbar \omega_{c}\left(n+\frac{1}{2}\right)+\frac{\hbar^{2} k_{z}^{2}}{2 m}
$$

These eigenvalues represent just the Landau's levels, but its solution (18a) is completely different from that one given by Landau on the variables " $x$ " and " $y$ ". One needs to point out that there is not displacement at all in the harmonic oscillation solution. Now, assuming a periodicity in the $z$-direction, $\Phi_{n, k_{z}}(\boldsymbol{x}, t)=\Phi_{n, k_{z}}\left(x, y, z+L_{z}, t\right)$, the usual condition $k_{z} L_{z}=2 \pi n^{\prime}, n^{\prime} \in \mathcal{Z}$ makes the eigenvalues to be written as and the general solution of Schrödinger's Equation (3) can be written as

$$
E_{n, n^{\prime}}=\hbar \omega_{c}(n+1 / 2)+\frac{\hbar^{2} 2 \pi^{2}}{m L_{z}^{2}} n^{\prime 2} .
$$

We must observe that these quantum numbers correspond to the degree of freedom in the " $y(n)$ " and " $z\left(n^{\prime}\right)$ " directions. The quantization condition of the magnetic flux appears rather naturally since $\mathrm{e}^{-i q B x y / \hbar c}=1$ for any " $x$ " and " $y$ " such that $q B x y / \hbar c=2 \pi j$, were $j \in \mathcal{Z}$. So, in particular one can ask this to happen for $x=L_{x}$ and $y=L_{y}$. Thus, it follows from the phase term that

$$
\frac{q B L_{x} L_{y}}{\hbar c}=2 \pi j, \quad j \in \mathcal{Z},
$$

where $B L_{x} L_{y}$ is the magnetic flux crossing the surface with area $L_{x} L_{y}$, and 
$\hbar c / q$ is the so-called quantum flux [26]. Then, expression (18a) is written as

$$
\Phi_{n n^{\prime} j}(\boldsymbol{x})=\frac{\sqrt{q B}}{\left(L_{y}^{2} L_{z}^{2} m c^{2} \hbar \omega_{c}\right)^{1 / 4}} e^{-i\left(\frac{2 \pi j}{L_{x} L_{y}} x y-\frac{2 \pi n^{\prime}}{L_{z}} z\right)} \psi_{n}\left(\frac{q B x}{\sqrt{m c^{2} \hbar \omega_{c}}}\right) .
$$

The degeneration of the eigenvalues (19) comes from the degree of freedom in " $X$ " and can be obtained by making use of the following quasi-classical argument: given the energy of the harmonic oscillator $E_{o}=\hbar \omega_{c}(n+1 / 2)$, we know the maximum displacement of the particle (classically) is given by $x_{\max }= \pm \sqrt{2 E_{o} / m \omega_{c}^{2}}$, and since the periodicity in the variable " $y$ " mentioned before is valid for any " $x$ " value, we must have that the maximum value of the quantum number " $\ddot{j}$ " must be

$$
\Delta j=\frac{q B L_{y}}{\pi \hbar c} x_{\max }=\frac{q B L_{y}}{\pi \hbar c} \sqrt{\frac{2 \hbar(n+1 / 2)}{m \omega_{c}}},
$$

and this represents the degeneration, $D(n)$, we have in the system

$$
D(n)=\left[\frac{q B L_{y}}{\pi \sqrt{m c^{2} \hbar \omega_{c}}} \sqrt{2 n+1}\right] .
$$

where $[\xi]$ means the integer part of the number $\xi$. Therefore, the general solution (absorbing the sign in the constants) is

$$
\begin{aligned}
\Psi(\boldsymbol{x}, t)= & \left.\sum_{n, n} \sum_{j=0}^{D(n)} C_{n n^{\prime} j} \sqrt{\frac{2 \pi j}{L_{x} L_{y}^{2} L_{z}}}\left(\frac{\hbar}{m \omega_{c}}\right)^{1 / 4} \mathrm{e}^{-i\left(\frac{2 \pi j}{L_{x} L_{y}} x y-\frac{2 \pi n^{\prime}}{L_{z}} z\right.}\right) \mathrm{e}^{-\frac{E_{n, n^{\prime}}}{\hbar} t} \\
& \times \psi_{n}\left(\sqrt{\frac{\hbar}{m \omega_{c}}}\left(\frac{2 \pi j}{L_{x} L_{y}}\right) x\right),
\end{aligned}
$$

where the constants $C_{n n^{\prime} j}$ must satisfy that $\sum_{n, n^{\prime}, j}\left|C_{n n^{\prime} j}\right|^{2}=1$. The Landau's levels $E_{n, n^{\prime}}$ are given by expression (19).

\section{The Analytical Approach for Case $B \perp E$}

This case is illustrated in Figure 2.

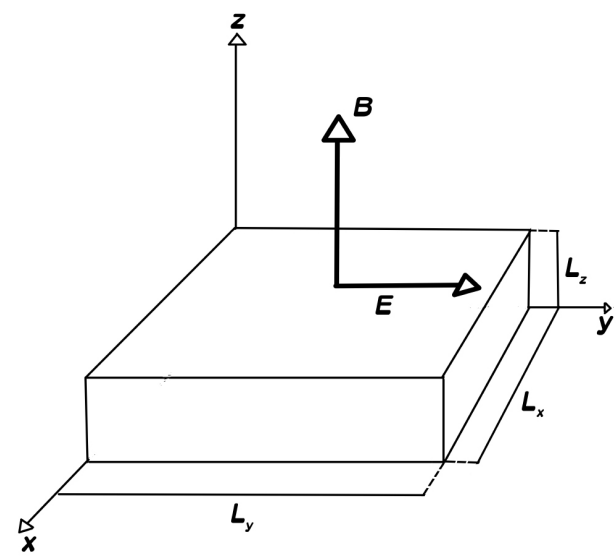

Figure 2. Electric charged in a flat box with magnetic and electric fields. 
Where the magnetic and electric constant fields are given by $\boldsymbol{B}=(0,0, B)$ and $\boldsymbol{E}=(0, \mathcal{E}, 0)$. We select Landau's gauge for the magnetic field such that the vector and scalar potentials are $\boldsymbol{A}=(-B y, 0,0)$ and $\phi=-\mathcal{E} y$. Then, our Hamiltonian is [21] [22] [23]

$$
\hat{H}=\frac{\left(\hat{\boldsymbol{p}}-\frac{q}{c} \boldsymbol{A}\right)^{2}}{2 m}+q \phi(\boldsymbol{x}),
$$

and the Schrödinger's equation,

$$
i \hbar \frac{\partial \Psi}{\partial t}=\hat{H} \Psi
$$

is written as

$$
i \hbar \frac{\partial \Psi}{\partial t}=\left\{\frac{1}{2 m}\left(\hat{p}_{x}+\frac{q B}{c} y\right)^{2}+\frac{\hat{p}_{y}^{2}}{2 m}+\frac{\hat{p}_{z}^{2}}{2 m}\right\} \Psi-q \mathcal{E} y \Psi .
$$

Using the definition $\hat{p}_{j}=-i \hbar \partial / \partial x_{j}$ and the commutation relation $\left[x_{k}, \hat{p}_{j}\right]=i \hbar \delta_{j} k$, the above expression is written as the following partial differential equation

$$
i \hbar \frac{\partial \Psi}{\partial t}=-\frac{\hbar^{2}}{2 m} \frac{\partial^{2} \Psi}{\partial x^{2}}-i \frac{q B \hbar}{m c} \frac{\partial \Psi}{\partial x}+\frac{q^{2} B^{2}}{2 m c^{2}} y^{2} \Psi-\frac{\hbar^{2}}{2 m} \frac{\partial^{2} \Psi}{\partial y^{2}}-\frac{\hbar^{2}}{2 m} \frac{\partial^{2} \Psi}{\partial z^{2}}-q \mathcal{E} y \Psi .
$$

Taking the Fourier transform, with respect the $x$-variable, $\hat{\Psi}(k, y, z, t)=\mathcal{F}_{x}[\Psi(\boldsymbol{x}, t)]$, the resulting expression is

$$
i \hbar \frac{\partial \hat{\Psi}}{\partial t}=\left[\frac{\hbar^{2} k^{2}}{2 m}-\left(\frac{q B \hbar k}{m c}+q \mathcal{E}\right) y+\frac{q^{2} B^{2}}{2 m c^{2}} y^{2}\right] \hat{\Psi}-\frac{\hbar^{2}}{2 m} \frac{\partial^{2} \hat{\Psi}}{\partial y^{2}}-\frac{\hbar^{2}}{2 m} \frac{\partial^{2} \hat{\Psi}}{\partial z^{2}} .
$$

By proposing a solution of the form

$$
\hat{\Psi}(k, y z, t)=\mathrm{e}^{-i E t / \hbar+i k_{z} z} \Phi(k, y)
$$

and after some rearrangements, the resulting equation for $\Phi$ is

$$
-\frac{\hbar^{2}}{2 m} \frac{\mathrm{d}^{2} \Phi}{\mathrm{d} y^{2}}+\frac{1}{2} m \omega_{c}^{2}\left(y-y_{0}\right)^{2} \Phi=E^{\prime} \Phi,
$$

where $\omega_{c}$ is the cyclotron frequency (13a), and we have made the definitions

$$
y_{0}=\frac{\hbar c}{q B} k+\frac{m c^{2} \mathcal{E}}{q B^{2}}
$$

and

$$
E^{\prime}=E-\frac{\hbar^{2} k^{2}}{2 m}-\frac{\hbar^{2} k_{z}^{2}}{2 m}+\frac{1}{2 m}\left(\hbar k+\frac{m c \mathcal{E}}{B}\right)^{2} .
$$

This equation is again the quantum harmonic oscillator on the variable " $y$ " with a cyclotron frequency $\omega_{c}$ and displaced by a quantity $y_{0}$. Therefore, the solution (14) is

$$
\Phi(k, y)=\psi_{n}\left(\sqrt{\frac{m \omega_{c}}{\hbar}}\left(y-y_{0}\right)\right)
$$


and

$$
E_{n}^{\prime}=\hbar \omega_{c}(n+1 / 2) .
$$

Thus, the solution in the Fourier space is

$$
\hat{\Psi}(k, y, z, t)=\mathrm{e}^{-i E_{n, k_{z}} t / \hbar+i k_{z} z} \psi_{n}\left(\sqrt{\frac{m \omega_{c}}{\hbar}}\left(y-y_{0}\right)\right)
$$

with the energies $E_{n, k_{z}}$ given by

$$
E_{n, k_{z}}=\hbar \omega_{c}(n+1 / 2)+\frac{\hbar^{2} k_{z}^{2}}{2 m}-\frac{m c^{2} \mathcal{E}^{2}}{2 B^{2}}-\frac{c \mathcal{E} \hbar}{B} k .
$$

The solution in the space-time is obtained by applying the inverse Fourier transformation,

$$
\Psi_{n, k_{z}}(\boldsymbol{x}, t)=\mathcal{F}\left[\hat{\Psi}_{n, k_{z}}(k, y, z, t)\right]=\frac{1}{\sqrt{2 \pi}} \int_{\Re} \mathrm{e}^{-i x k} \hat{\Psi}_{n, k_{z}}(k, y, z, t) \mathrm{d} k,
$$

which after a proper change of variable and rearrangements, we get the normalized function (ignoring the sign)

$$
\Psi_{n, k_{z}}(\boldsymbol{x}, t)=\frac{\sqrt{q B}}{\left(L_{y}^{2} L_{z}^{2} m c^{2} \hbar \omega_{c}\right)^{1 / 4}} \mathrm{e}^{-i \phi_{n, k_{z}}(x, t)} \psi_{n}\left(\frac{q B}{\sqrt{m c^{2} \hbar \omega_{c}}}\left(x-\frac{c \mathcal{E} t}{B}\right)\right),
$$

where the phase $\phi_{n, k_{z}}(x, t)$ has been defined as

$$
\begin{aligned}
\phi_{n, k_{z}}(\boldsymbol{x}, t)= & {\left[\hbar \omega_{c}(n+1 / 2)+\frac{\hbar^{2} k_{z}^{2}}{2 m}-\frac{m c^{2} \mathcal{E}^{2}}{2 B^{2}}\right] \frac{t}{\hbar} } \\
& -k_{z} z+\frac{q B}{\hbar c}\left(x-\frac{c \mathcal{E} t}{B}\right)\left(y-\frac{m c^{2} \mathcal{E}}{q B^{2}}\right) .
\end{aligned}
$$

asking for the periodicity with respect to the variable " $z$ ", $\Psi_{n, k_{z}}(x, t)=\Psi_{n, k_{z}}\left(z, y, z+L_{z}, t\right)$, it follows that $k_{z} L_{z}=2 \pi n^{\prime}$ where $n^{\prime}$ is an integer number, and the above phase is now written as

$$
\begin{aligned}
\phi_{n n^{\prime}}(\boldsymbol{x}, t)= & {\left[\hbar \omega_{c}(n+1 / 2)+\frac{\hbar^{2} 2 \pi^{2} n^{\prime 2}}{m L_{z}^{2}}-\frac{m c^{2} \mathcal{E}^{2}}{2 B^{2}}\right] \frac{t}{\hbar}-\frac{2 \pi n^{\prime}}{L_{z}} z } \\
& +\frac{q B}{\hbar c}\left(x-\frac{c \mathcal{E} t}{B}\right)\left(y-\frac{m c^{2} \mathcal{E}}{q B^{2}}\right) .
\end{aligned}
$$

Note from this expression that the term $\mathrm{e}^{-i \phi(x, t)}$ contains the element $\mathrm{e}^{i \frac{q B}{\hbar c} x y}$, and by assuming the periodic condition $\Psi(x, t)=\Psi\left(x, y+L_{y}, z, t\right)$, will imply that $\Psi(\boldsymbol{x}, t)$ will be periodic with respect to the variable " $y$ ", for any " $x$ " at any time " $t$ ". In particular, this will be true for $x=L_{x}$. This last assumption brings about the quantization of the magnetic flux of the form

$$
\frac{q B L_{x} L_{y}}{\hbar c}=2 \pi j, \quad J \in \mathcal{Z},
$$

obtaining the same expression as (20), and this phase is now depending on the quantum number “ $\rho$ " 


$$
\phi_{n n^{\prime} j}(\boldsymbol{x}, t)=e_{n n^{\prime}} t / \hbar-\frac{2 \pi n^{\prime}}{L_{z}} z+\frac{2 \pi j}{L_{x} L_{y}} x y-\frac{2 \pi j}{L_{x} L_{y}}\left[\frac{m c^{2} \mathcal{E}}{q B^{2}} x+\frac{c \mathcal{E}}{B} t y\right]
$$

where $e_{n n^{\prime}}$ is the energy associated with the system,

$$
e_{n, n^{\prime}}=\hbar \omega_{c}(n+1 / 2)+\frac{2 \pi^{2} \hbar^{2}}{m L_{z}^{2}} n^{\prime 2}+\frac{m c^{2} \mathcal{E}^{2}}{2 B^{2}} .
$$

In this way, from these relations and the expression (39) we have a family of solutions $\left\{\Psi_{n n^{\prime} j}(x, t)\right\}_{n, n^{\prime}, j \in \mathcal{Z}}$ of the Schrödinger Equation (27),

$$
\Psi_{n n^{\prime} j}(\boldsymbol{x}, t)=\sqrt{\frac{2 \pi j}{L_{x} L_{y}^{2} L_{z}}}\left(\frac{\hbar}{m \omega_{c}}\right)^{1 / 4} \mathrm{e}^{-i \phi_{n n^{\prime} j}(\boldsymbol{x}, t)} \psi_{n}\left(\sqrt{\frac{\hbar}{m \omega_{c}}}\left(\frac{2 \pi j}{L_{x} L_{y}}\right)\left(x-\frac{c \mathcal{E} t}{B}\right)\right),
$$

Now, by the same arguments we did in the previous case, the degeneration of the systems would be given by (23), and the general solution would be of the form

$$
\Psi(\boldsymbol{x}, t)=\sum_{n, n^{\prime}} \sum_{j=0}^{D(n)} \tilde{C}_{n n^{\prime} j} \Psi_{n n^{\prime} j}(\boldsymbol{x}, t) .
$$

\section{The Analytical Approach for Case $B \| E$}

Figure 3 shows this case.

The fields are of form $\boldsymbol{B}=(0, B, 0)$ and $\boldsymbol{E}=(0, \mathcal{E}, 0)$. The scalar and vector potentials are chosen as $\boldsymbol{A}=(B z, 0,0)$ and $\phi=-\mathcal{E} y$. The Shrödinger equation is for this case as

$$
i \hbar \frac{\partial \Psi}{\partial t}=\left\{\frac{\left(\hat{p}_{x}-q B z / c\right)^{2}}{2 m}+\frac{\hat{p}_{y}^{2}}{2 m}+\frac{\hat{p}_{z}^{2}}{2 m}-q \mathcal{E} y\right\} \Psi,
$$

which defines the following partial differential equation

$$
\begin{aligned}
i \hbar \frac{\partial \Psi}{\partial t}= & -\frac{\hbar^{2}}{2 m} \frac{\partial^{2} \Psi}{\partial x^{2}}+i \frac{q B \hbar z}{m c} \frac{\partial \Psi}{\partial x}+\frac{q^{2} B^{2}}{2 m c^{2}} z^{2} \Psi \\
& -\frac{\hbar^{2}}{2 m} \frac{\partial^{2} \Psi}{\partial y^{2}}-\frac{\hbar^{2}}{2 m} \frac{\partial^{2} \Psi}{\partial z^{2}}-q \mathcal{E} y \Psi .
\end{aligned}
$$

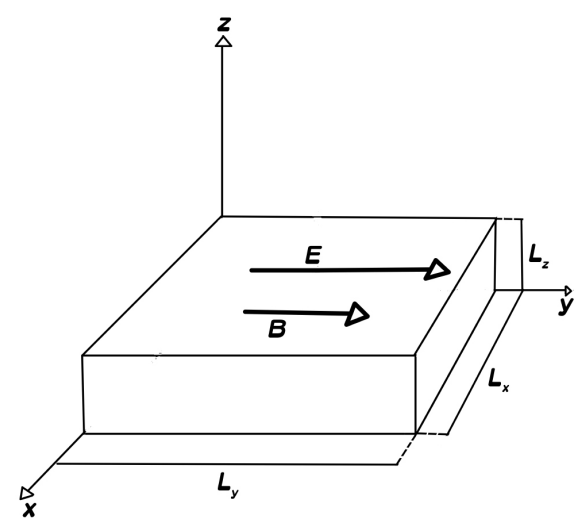

Figure 3. Electric charged in a flat box with parallel electric and magnetic fields. 
Proposing a solution of the form $\Psi(x, t)=\mathrm{e}^{-i E t / \hbar} \Phi(\boldsymbol{x})$, we get the following eigenvalue problem

$$
\begin{aligned}
E \Phi= & -\frac{\hbar^{2}}{2 m} \frac{\partial^{2} \Phi}{\partial x^{2}}+i \frac{q B \hbar z}{m c} \frac{\partial \Phi}{\partial x}+\frac{q^{2} B^{2}}{2 m c^{2}} z^{2} \Phi \\
& -\frac{\hbar^{2}}{2 m} \frac{\partial^{2} \Phi}{\partial y^{2}}-\frac{\hbar^{2}}{2 m} \frac{\partial^{2} \Phi}{\partial z^{2}}-q \mathcal{E} y \Phi .
\end{aligned}
$$

Applying the Fourier transform over the $x$-variable, $\hat{\Phi}(k, y, z)=\mathcal{F}_{x}[\Phi(\boldsymbol{x})]$, the following equation arises after some rearrangements

$$
E \hat{\Phi}=\frac{(\hbar k+q B z / c)^{2}}{2 m} \hat{\Phi}-\frac{\hbar^{2}}{2 m} \frac{\partial^{2} \hat{\phi}}{\partial z^{2}}-\frac{\hbar^{2}}{2 m} \frac{\partial^{2} \hat{\Phi}}{\partial y^{2}}-q \mathcal{E} y \hat{\Phi},
$$

which can be written as

$$
-\frac{\hbar^{2}}{2 m} \frac{\partial^{2} \hat{\Phi}}{\partial z^{2}}+\frac{1}{2} m \omega_{c}\left(z+z_{0}\right)^{2} \hat{\Phi}-\frac{\hbar^{2}}{2 m} \frac{\partial^{2} \hat{\Phi}}{\partial y^{2}}-q \mathcal{E} y \hat{\Phi},
$$

where $\omega_{c}$ is the cyclotron frequency (13a), and $z_{0}=z_{0}(k)$ has been defined as

$$
z_{0}=\frac{\hbar c}{q B} k
$$

This equation admits a variable separable approach since by the proposition $\hat{\Phi}(k, y, z)=f(k, z) g(y)$, the following equations are bringing about

$$
-\frac{\hbar^{2}}{2 m} \frac{\mathrm{d}^{2} f}{\mathrm{~d} z^{2}}+\frac{1}{2} m \omega_{c}^{2}\left(z+z_{0}\right)^{2}=E^{(1)} f
$$

and

$$
-\frac{\hbar^{2}}{2 m} \frac{\mathrm{d}^{2} g}{\mathrm{~d} y^{2}}-g \mathcal{E} y g=E^{(2)} g,
$$

where $E=E^{(1)}+E^{(2)}$. The solutions of these equations are, of course, the quantum harmonic oscillator and the quantum bouncer, which are given by

$$
f_{n}(k, z)=A_{n} \mathrm{e}^{-\xi^{2} / 2} H_{n}(\xi), \quad \xi=\sqrt{\frac{m \omega_{c}}{\hbar}}\left(z+z_{0}\right), \quad E_{n}^{(1)}=\hbar \omega_{c}(n+1 / 2) .
$$

and

$$
g_{n^{\prime}}(y)=\frac{A i\left(\tilde{\xi}-\tilde{\xi}_{n^{\prime}}\right)}{\left|A i^{\prime}\left(-\tilde{\xi}_{n^{\prime}}\right)\right|}, \quad \tilde{\xi}=y / l, \quad E_{n}^{(2)}=-q \mathcal{E} l \tilde{\xi}_{n^{\prime}},
$$

where $A_{n}=\left(m \omega_{c} / \pi \hbar\right)^{1 / 4} / \sqrt{2^{n} n !}, \quad l=\left(\hbar^{2} /(-2 m q \mathcal{E})\right)^{1 / 3}, \quad A i\left(-\tilde{\xi}_{n^{\prime}}\right)=0$, and $A i^{\prime}(\xi)$ is the differentiation of the Airy function. In this way, we have

$$
\begin{aligned}
& \hat{\Phi}_{n, n^{\prime}}(k, y, z)=a_{n^{\prime}} \psi_{n}\left(\sqrt{\frac{m \omega_{c}}{\hbar}}\left(z+z_{0}\right)\right) A i\left(l^{-1}\left(y-y_{n^{\prime}}\right)\right), \\
& E_{n, n^{\prime}}=\hbar \omega_{c}(n+1 / 2)-q \mathcal{E} y_{n^{\prime}},
\end{aligned}
$$

where we have defined $a_{n^{\prime}}$ as $a_{n^{\prime}}=1 /\left|A i^{\prime}\left(-l^{-1} y_{n^{\prime}}\right)\right|$. Now, the inverse Fourier transformation will affect only the quantum harmonic oscillator function $\psi_{n}$ through the $k$-dependence on the parameter $z_{0}$, and the resulting expression is 


$$
\Phi_{n, n^{\prime}}(x)=\frac{a_{n^{\prime}} \sqrt{q B}}{\left(m c^{2} \hbar \omega_{c}\right)^{1 / 4}} \mathrm{e}^{i \frac{q B}{\hbar c} x z} \psi_{n}\left(\frac{q B x}{\sqrt{m c^{2} \hbar \omega_{c}}}\right) A i\left(l^{-1}\left(y-y_{n^{\prime}}\right)\right) .
$$

Now, asking for the periodicity condition of the above solution with respect the z-variable, $\Phi_{n, n^{\prime}}(\boldsymbol{x})=\Phi_{n, n^{\prime}}\left(x, y, z+L_{z}\right)$, the periodicity must satisfy for any $\mathrm{x}$-values, and in particular for $x=L_{x}$. Thus it follows the quantization expression for the magnetic flux

$$
\frac{q B L_{x} L_{z}}{\hbar c}=2 \pi j, \quad j \in \mathcal{Z} .
$$

Using the same arguments shown above for the degeneration of the system, we have the same expression (23) for the degeneration of the system and the function (55) is given by (normalized)

$$
\begin{aligned}
\Phi_{n n^{\prime} j}(\boldsymbol{x})= & a_{n^{\prime}} \sqrt{\frac{2 \pi j}{L_{x} L_{y}^{2} L_{z}}}\left(\frac{\hbar}{m \omega_{c}}\right)^{1 / 4} \mathrm{e}^{i \frac{2 \pi j}{L_{x} L_{z}} x z} \psi_{n}\left(\sqrt{\frac{\hbar}{m \omega_{c}}}\left(\frac{2 \pi j}{L_{x} L_{y}}\right) x\right) \\
& \times A i\left(l^{-1}\left(y-y_{n^{\prime}}\right)\right) .
\end{aligned}
$$

Then, we have obtained a family of solution of the Schrödinger Equation (48),

$$
\Psi_{n, n^{\prime}}(\boldsymbol{x}, t)=\mathrm{e}^{-i E_{n, n^{\prime}} / \hbar} \Phi_{n n^{\prime} j}(\boldsymbol{x}),
$$

where the energies $E_{n, n^{\prime}}$ are given by the expression (54). The general solution of (48) can be written as

$$
\Psi(\boldsymbol{x}, t)=\sum_{n, n^{\prime}} \sum_{j=0}^{D(n)} C_{n, n^{\prime}}^{*} \mathrm{e}^{-i E_{n, n^{\prime}} / \hbar} \mathrm{e}^{i \frac{2 \pi j}{L_{x} L_{z}} x z} \tilde{u}_{n, n^{\prime}}(x, y),
$$

with the condition $\sum_{n, n^{\prime}}\left|C_{n, n^{\prime}}^{*}\right|^{2}=1$, and where it has been defined the functions $\tilde{u}_{n, n^{\prime}}$ as

$$
\begin{aligned}
\tilde{u}_{n, n^{\prime}}(x, y)= & a_{n^{\prime}} \sqrt{\frac{2 \pi j}{L_{x} L_{y}^{2} L_{z}}}\left(\frac{\hbar}{m \omega_{c}}\right)^{1 / 4} \psi_{n}\left(\sqrt{\frac{\hbar}{m \omega_{c}}}\left(\frac{2 \pi j}{L_{x} L_{y}}\right) x\right) \\
& \times A i\left(l^{-1}\left(y-y_{n^{\prime}}\right)\right) .
\end{aligned}
$$

\section{Conclusion and Comment}

We have studied the quantization of a charged particle in a flat box and under constant magnetic and electric fields for several electromagnetic static cases using Fourier transformation to solve the linear differential equations resulting from the Shrödinger's equation, and we have shown that the full solution obtained is different from Landau's solution for the wave function, but as expected, Landau's levels appear as the solution of the eigenvalues. In all cases, a characteristic phase appears which helps us to find the quantization of the magnetic flux in a very natural way. We consider that the approach given here could be very useful to understand quantum Hall effect and related phenomena mainly because with Landau' solution a Hall's voltage appears (which is not possible with Landau' solution due to free particle solution on this direction). In addition, the 
resulting degeneration in our calculations is different, and this one is used in the Fermi-Dirac distribution function to calculate the axial and transversal conductivities on the Hall's experiments.

\section{Conflicts of Interest}

The authors declare no conflicts of interest regarding the publication of this paper.

\section{References}

[1] Klitzing, K.V., Dorda, G. and Pepper, M. (1980) Physical Review Letters, 45, 494. https://doi.org/10.1103/PhysRevLett.45.494

[2] Landau, L.D. and Lifshitz, E.M. (2013) Quantum Mechanics: Non-Relativistic Theory, Volume 3. Elsevier, Amsterdam.

[3] Ando, T., Matsumoto, Y. and Uemura, Y. (1975) Journal of the Physical Society of Japan, 39, 279-288. https://doi.org/10.1143/JPSJ.39.279

[4] Laughlin, R.B. (1981) Physical Review B, 23, 5632. https://doi.org/10.1103/PhysRevB.23.5632

[5] Halperin, B.I. (1982) Physical Review B, 25, 2185. https://doi.org/10.1103/PhysRevB.25.2185

[6] Laughlin, R.B. (2000) Uspekhi Fizicheskikh Nauk, 170, 292-303. https://doi.org/10.3367/UFNr.0170.200003f.0292

[7] Tsui, D.C., Stormer, H.L. and Gossard, A.C. (1982) Physical Review Letters, 48, 1559. https://doi.org/10.1103/PhysRevLett.48.1559

[8] Laughlin, R.B. (1983) Physical Review Letters, 50, 1395-1398. https://doi.org/10.1103/PhysRevLett.50.1395

[9] Jain, J.K. (1989) Physical Review Letters, 63, 199-202. https://doi.org/10.1103/PhysRevLett.63.199

[10] Bernevig, B.A., Hughes, T.L. and Zhang, S.-C. (2006) Science, 314, 1757-1761. https://doi.org/10.1126/science.1133734

[11] König, M., Wiedmann, S., Brüne, C., Roth, A., Buhmann, H., Molenkamp, L.W., Qi, X.-L. and Zhang, S.-C. (2007) Science, 318, 766-770. https://doi.org/10.1126/science.1148047

[12] Li, J., Chu, R.-L., Jain, J.K. and Shen, S.-Q. (2009) Physical Review Letters, 102, Article ID: 136806. https://doi.org/10.1103/PhysRevLett.102.136806

[13] Jiang, H., Wang, L., Sun, Q.-F. and Xie, X.C. (2009) Physical Review B, 80, Article ID: 165316. https://doi.org/10.1103/PhysRevB.80.165316

[14] Groth, C.W., Wimmer, M., Akhmerov, A.R., Tworzydlo, J. and Beenakker, C.W.J. (2009) Physical Review Letters, 103, Article ID: 196805. https://doi.org/10.1103/PhysRevLett.103.196805

[15] Mong, R.S.K., Essin, A.M. and Moore, J.E. (2010) Physical Review B, 81, Article ID: 245209. https://doi.org/10.1103/PhysRevB.81.245209

[16] Pesin, D. and Balents, L. (2010) Nature Physics, 6, 376-381. https://doi.org/10.1038/nphys1606

[17] Fu, L. and Kane, C.L. (2008) Physical Review Letters, 100, Article ID: 096407. https://doi.org/10.1103/PhysRevLett.100.096407

[18] Freedman, M.H., Larsen, M. and Wang, Z.H. (2002) Communications in Mathe- 
matical Physics, 227, 605-622. https://doi.org/10.1007/s002200200645

[19] Kitaev, A. (2006) Annals of Physics, 321, 2-111.

https://doi.org/10.1016/j.aop.2005.10.005

[20] Prange, R.E. (1981) Physical Review B, 23, 4802-4805.

https://doi.org/10.1103/PhysRevB.23.4802

[21] Laughlin, R.B. (1999) Reviews of Modern Physics, 71, 863-874.

https://doi.org/10.1103/RevModPhys.71.863

[22] Yoshioka, D. (2013) The Quantum Hall Effect, Volume 133. Springer Science \& Business Media, Berlin.

[23] Datta, S. (1997) Electronic Transport in Mesoscopic Systems. Cambridge University Press, Cambridge.

[24] Messiah, A. (1999) Quantum Mechanics. Dover, Mineola.

[25] Rudin, W. (1974) Functional Analysis. TATA McGraw-Hill Publishing Co., New Delhi.

[26] Deaver Jr., B.S. and William, F.M. (1961) Physics Review Letters, 7, 43-46. https://doi.org/10.1103/PhysRevLett.7.43 